\title{
Locating Station of One-Way Carsharing Based on Spatial Demand Characteristics
}

\author{
Xiaohong Chen, ${ }^{1}$ Jiaqi Cheng $\mathbb{D}^{1},{ }^{1}$ Jianhong Ye $\mathbb{D},{ }^{1}$ Yong $\mathrm{Jin}^{2}{ }^{\mathrm{Xi}} \mathrm{Li},{ }^{2}$ and Fei Zhang ${ }^{2}$ \\ ${ }^{1}$ Key Laboratory of Road and Traffic Engineering of the Ministry of Education, Tongji University, 4800 Caoan Road, \\ Shanghai 201804, China \\ ${ }^{2}$ Global Carsharing \& Rental Co., Ltd., 888 South Moyu Road, Shanghai 201805, China \\ Correspondence should be addressed to Jianhong Ye; yjh1875@hotmail.com
}

Received 16 December 2017; Revised 26 March 2018; Accepted 15 April 2018; Published 23 May 2018

Academic Editor: Emanuele Crisostomi

Copyright (C) 2018 Xiaohong Chen et al. This is an open access article distributed under the Creative Commons Attribution License, which permits unrestricted use, distribution, and reproduction in any medium, provided the original work is properly cited.

\begin{abstract}
This study aims to explore factors that affect carsharing demand characteristics in different time periods based on EVCARD transaction data, which is the largest station-based one-way carsharing program in Shanghai, China. Monthly usage intensity and degree of usage imbalance are used as proxies of demand. This study uses three groups of independent variables: carsharing station attributes, built environment (density, diversity, design, and destination accessibility), and transportation facilities. The adaptive elastic net regression is developed to identify factors that influence carsharing usage intensity and degree of usage imbalance after factor selection using extra-randomized-tree algorithm. Finally, a station layout is proposed according to both usage intensity and degree of imbalance. The main results of this study are presented as follows: (1) different effects of built environment and transportation factors cause dynamic demand across different time periods; (2) factors with positive and negative effect on carsharing demand are divided clearly for guidance of the carsharing station layout; (3) public parking space leads to more personal vehicle trip compared to a carsharing trip; and (4) as public transportation, the relationship of the metro and carsharing is complementary. However, the bus stop and carsharing have a competitive relationship. This study provides a carsharing layout method based on both usage intensity and degree of imbalance. Furthermore, several policies concerning carsharing are proposed.
\end{abstract}

\section{Introduction}

Carsharing service allows users to avoid the money and time cost of car ownership, such as maintenance, insurance, and finding parking space. It offers more flexible car travel [1] and has been promptly developed in recent years. Many previous studies have shown that carsharing service can effectively reduce vehicle ownership [2,3], reduce the vehicle miles traveled $[4,5]$, and control the greenhouse effect $[6$, 7], particularly when some carsharing uses electric vehicles [8]. Carsharing can improve social equality and provide a convenient trip option to people who cannot afford a car [9]. These benefits for the society rely on the scale of the carsharing program and the program sustainability, which are dependent on the profit and cost situation of carsharing.

The mode of operation is primarily considered based on profit and cost from the demand feature situations.
Carsharing has two operation categories, namely, stationbased carsharing (SBC) and free-floating carsharing (FFC). Moreover, there are two types of SBC. The first type requires returning the rented vehicle to the same station, which is called round-trip carsharing (RTC). The second type allows users to drop off the vehicle at any station after finishing their trip, which is called one-way carsharing (OWC). However, FFC allows users to pick up and drop off the vehicle at any location within a specified service area.

In RTC, users eventually drop off vehicles to the pick-up station, which does not require dispatch in normal operation. OWC improves the flexibility of using the vehicle compared to RTC while bringing extra operational burden. Given that dropping off at another station is allowed, there is a high probability of a spatial imbalance over the stations. The operator needs to arrange the vehicle relocation to maintain regular operation, which results in increased operation cost. 
OWC and FFC allow one-way trip, which creates the problem of spatiotemporal imbalance of demand. This problem can be handled using different approaches. FFC can only maintain system balance by dispatching techniques. However, OWC is a station-based system; therefore, it can mitigate the problem of demand imbalance by appropriate location of stations. Supposing that there is a more demandbalanced area, the pick-up and drop-off at a station can be counteracted during a given temporal interval. Therefore, the layout of stations in the more balanced areas can strategically reduce vehicle relocation. In the long term, it can reduce dispatching cost. Therefore, it is essential to find a smallscale area with more balanced demand in OWC. However, the authors found that studies focusing on this issue are rare. Thus, this research will address this gap in literature.

Given that station usage intensity is related to the operator's operational profits and station usage imbalance is related to operational cost, finding areas with higher carsharing usage intensity but lower degree of station usage imbalance is significantly important to the station-based OWC program. Therefore, this research uses station usage intensity and degree of station usage imbalance as proxies to represent the demand characteristics to explore how factors affect the carsharing demand characteristics in different time period of the day.

Section 2 reviews the literature related to the identification of factors affecting the demand characteristics in the carsharing field. Section 3 describes the data profile and develops the monthly usage intensity model and usage imbalance model. Section 4 presents the result of the models and then discusses the factors affecting the demand characteristics of carsharing and provides result of station layout. Finally, Section 5 provides a summary and the limitations of this study.

\section{Literature Review}

The research on carsharing has increased rapidly since 2010 [10]. The current document mainly contains seven subfields: the users' attitude and behavior, demand analysis, depot location, vehicle relocation, benefit evaluation, business models, and policy. Two issues are included in the demand analysis: (1) user characteristics or potential demand and (2) identification of factors that influence the current demand. This work aims to address the second issue.

Table 1 shows the current references based on analysis units, methods, source of variable data, dependent variables, and the division stage according to the operating model.

The current references mainly focus on RTC [11-16] and FFC [17-22], but the study on OWC is insufficient. In basic analysis unit, the user is always selected for the RTC model [11-13]. The spatial scales of a single station, the station cluster to land parcel (including grid, census tracts, transportation analysis zone, and district), are in increasing order [14-24]. The smaller the spatial scale of the analysis unit is, the greater the model can reflect the demand characteristics of the local space. However, the negative effect is the larger proportion of unexplained variance [23]. When the amount of data can ensure that the proportion of random change cannot obviously affect the model fitting, the analysis should be conducted using smaller space scale. The study using vehicle as an analysis unit mainly focuses on analyzing the usage rate of different types of vehicles [9].

Given the privacy protection and business sensitivity, early researchers cannot obtain operational data but discontinuously collect some sparse data $[14,15]$. These research studies assume that operators can dispatch a vehicle fully according to demand, where the vehicle number within the space unit can be used as proxy of the demand in that spatial unit [23]. Studies based on transaction data and operation record $\log$ increased. However, given that the demand is restrained by insufficient supply, the study on the demand can only be conducted using actual usage as proxy of the demand, which is more reliable than studies using supply as proxy for demand.

Dependent variables can be divided into usage frequency and usage duration, which are common dimensions of demand characteristics. Most studies take usage frequency and its various variants (such as vehicle usage rate) as dependent variables. These studies take booking quantity as usage frequency $[9,11-13,15,16,19,21]$ but do not consider pick-up and drop-off mainly because the pick-up and dropoff of RTC are in the same station. On the contrary, there is no station in FFC. In OWC, the station not only generates carsharing trip but also is attracting it as well. Completing a carsharing trip based on two stations requires a separate pick-up and drop-off. In addition, vehicles of RTC belong to one station; therefore, the effects of supply on demand can be eliminated by controlling the variable of the vehicle number. The vehicle usage rate can be used as dependent variable [14]. Without station in FFC, one method to obtain usage frequency is by calculating the bookings in area unit, which makes booking density the most common variable in FFC $[17,20-22]$ including usage duration. However, no study has been found on station usage imbalance.

Only few research studies use a simple method of descriptive statistics [11] similar to the method used in this study. Most studies directly use multiple linear models $[12,14-17,19,23,24]$, while other studies exchange natural logarithms for dependent variables to make them in normal distribution [23]. Given that dependent variables meet with other exponential family distribution, the generalized linear model is used [12]. Moreover, given that an independent variable has large zero value, the zero-inflated model $[13,20]$ is used. The Dynamic Ordered Probit Model is used because decisions of multiple frequencies of usage are cumulatively related to each other [13].

In summary, the research on station-based OWC is rare, and this work will focus on this operational mode. Moreover, for the dependent variable, the station usage intensity used in this study comprehensively considers the demands for pick-up and drop-off. However, the degree of station usage imbalance has not yet been studied. Finally, the analysis of demand characteristics across different time period is not sufficient in the current literature. In this study, time is divided into several parts, and each time section of the day is modeled individually. 
TABLE 1: Summary of document on identification of factors affecting demand characteristics.

\begin{tabular}{|c|c|c|}
\hline Item & Category & Literature \\
\hline \multirow{3}{*}{ Operational mode } & RTC & {$[11-16]$} \\
\hline & FFC & {$[17-22]$} \\
\hline & NEM (not explicitly mentioned) & {$[9,23,24]$} \\
\hline \multirow{6}{*}{ Basic analysis unit } & User (RTC) & [11-13] \\
\hline & Single station (RTC) & {$[14]$} \\
\hline & Station cluster (NEM) & {$[23]$} \\
\hline & Location $(\mathrm{FFC})$ & {$[19,20]$} \\
\hline & Land parcel (RTC, FFC, and NEM) & {$[15-18,21,22,24]$} \\
\hline & Vehicle (NEM) & {$[9]$} \\
\hline \multirow{2}{*}{ Data sources of dependent variable } & Collected by authors & {$[14,15]$} \\
\hline & Transaction data or operation log & {$[9,11-13,16-24]$} \\
\hline \multirow{8}{*}{ Dependent variable } & Usage frequency [RTC and FFC] & {$[11-13,16,19,21]$} \\
\hline & Total vehicle hour travel [RTC] & {$[12]$} \\
\hline & Monthly hours per vehicle [RTC] & {$[14]$} \\
\hline & Vehicle usage rate [RTC and NEM] & {$[9,15]$} \\
\hline & Booking density $[\mathrm{FFC}]$ & {$[17,20-22]$} \\
\hline & Number of vehicles [NEM] & [24] \\
\hline & Vehicle unused duration [FFC] & {$[18]$} \\
\hline & Usage hours of station [NEM] & {$[23]$} \\
\hline \multirow{6}{*}{ Method } & Descriptive statistics & {$[11]$} \\
\hline & Generalized linear model & {$[9,12,20,21]$} \\
\hline & Multiple linear model & {$[12,14-17,19,23,24]$} \\
\hline & Dynamic ordered probit model & {$[13]$} \\
\hline & Duration model technique & {$[18]$} \\
\hline & Hot spots by ArcGIS & {$[22]$} \\
\hline \multicolumn{2}{|c|}{ Develop individually models for several time periods of the day } & {$[15,21,22]$} \\
\hline
\end{tabular}

\section{Data and Methodology}

3.1. Data Preparation. The four data sources used in this study are shown in Figure 3. The first data source is provided by the Shanghai EVCARD, which includes station information and transaction data starting from January 2015 to December 2016. EVCARD is the largest carsharing program in China. It is a station-based one-way system, which allows users to drop off car at any station after a trip. All vehicles in operation are Pure Battery Electric Vehicles (PBEVs), and the price is only based on the trip duration. The usage procedure is completely self-service. Station information includes station coordinates, station age (number of months from the start of operation until December 2016), parking space, if a station is located at an underground garage (this is related to whether or not the station is easy to find), and if a station has limited accessibility because it is located at an internal location of companies or government department where nonemployees are not allowed to enter for a specific time period or permanently. Other stations nearby can be calculated based on the coordinates data using Geographic Information System (GIS) software. A station includes several parking lots (from two to dozens). Transaction data includes membership number, vehicle license plate number, time and station name for pick-up and drop-off, trip duration, fee, deductions of the booking fee, and the reason for the reduction. Given that the data are commercially sensitive, these data are only permitted to display directly a limited part of the operator request.

The second data source is composed of Point of Interest (POI) data in Shanghai, which was collected through AMAP API in November 2016. AMAP is a Google-Maps-like E-map, which is popular and widely used in China. The total collected POIs reached 427210, which are categorized into eleven types based on land use attributes: residential area, culture places, business, medical service, public authority, social and recreation, shopping, college and university, industry, transportation, and intersections. Among these types, college and university is used instead of a larger type of education because, in addition to being found in preresearch, other educational areas, such as middle school, primary school, kindergarten, and training institution, have no statistically significant effect on the demand characteristics. However, college and university plays an important role; therefore, it replaces the educational type. The transportation type is very important; therefore, it is separately categorized into more specific subtypes, including airport, train station, intercity coach station, metro station, bus stop, and traditional car rental station. Intersection POIs are used to represent the street design. Moreover, POI-mixed entropy is calculated 
based on POIs, which refers to the entropy form of research [25].

The third data source is part of the latest census data of Shanghai, which was collected in 2010 by the Shanghai Municipal Bureau of Statistics. Resident density, job density, and average trip distance of each block were used in this research. Average trip distance is used to represent the accessibility of a destination.

Finally, the fourth data source is the information of road network in Shanghai, which includes freeway, arterial road, secondary road, local street, one-way road, and two-way road. We counted the length of each type of road in spatial unit to represent characteristics of local road network.

The four data sources are reorganized into three groups of features. The first feature is the station's attributes $(S)$. The second feature is the transportation facilities. The number of each type of transportation facilities located within the buffer of the carsharing station is used to present the transportation environment within the spatial unit. The third group of features refers to the $5 D$ principle of the built environment $[26,27]$. However, the fifth " $D$ " (distance to transit) is presented as the distance between a residential place or work place to the nearest public transportation station, which is not appropriate for this research. Therefore, we use the transportation facilities instead of the "distance to transit." Thus, independent variables shall be categorized and grouped as " $S+T+4 D$." The descriptive statistics of features and targets are summarized in Table 2 .

For sample selection, stable operational stations are selected in this study. Many stations have short operation period with data that ended on 10 December 2016. These stations are not well known to users; therefore, they are not included in this research because of their unstable performance. Data indicate that the use of vehicles becomes relatively stable when the station is operated into the fourth month (the booking changing rate is about 0.5 . The booking changing rate reaches 7.1 in the second month and 0.78 in the third month). In this research, we only include stations with more than three months of operation, while the booking changing rate is less than 0.3 in three consecutive months from October to December 2016. These stations are judged as relatively stable. Eventually, 551 stations participated in this research. Figure 1 shows the spatial distribution of all stations operating in Shanghai as well as the stations participating in this research. The proportion of stations participating in this research is the same as the proportion of the whole station at each area. The central areas have less than $4 \%$ stations and parking spaces, while $95 \%$ of the stations are located at the outskirts area. We divided Shanghai into a hexagonal grid, where the area of the hexagonal spatial unit is $2 \mathrm{~km}^{2}$ (similar to a circle with 800 -meter radius in the area). The stations participating in this research are assigned to this hexagon unit based on spatial location. The hexagonal spatial unit is used as an analysis unit in this research.

Since the EVCARD station, as basic object in this research, is selected according to the fact that bookings changing rate is relatively stable during the three months from November to December 2016, the dependent variable in this research also requires the relative stability of station operation performance, where the collecting time of POI data shall be within this time range. Therefore, only the threemonth transaction data from October to December 2016 is investigated, which covers about $40 \%$ of the total bookings.

3.1.1. Temporal Dynamics of Demand. To investigate the temporal dynamics of demand, the data is divided into two subsets: workday and nonworkday. In China, given that adjustment is made when national holidays overlap weekdays, some weekends become workdays and some workdays become holidays. Therefore, workday and nonworkday are used to separate time instead of weekday and weekend.

Based on these conditions, the two subsets of data are divided into five time sections of the day, which are as follows according to the trip temporal distribution of the EVCARD (Figure 2): early morning (EM) (2:00-6:00, $4 \mathrm{~h})$, morning peak (MP) (6:00-10:00, 4h), off-peak (OP) (10:00-16:00, $6 \mathrm{~h})$, evening peak (EP) (16:00-20:00, $4 \mathrm{~h})$, and night (NT) (20:00-2:00, 6h). Given the few bookings in EM which are quite random, the later four time sections are included in this research. Finally, eight time sections were obtained, and these time sections are mathematically expressed as follows:

$$
T=\left\{T_{a}^{b} \mid a \in A, b \in B\right\},
$$

where $A=\{$ workday, nonworkday $\}$ and $B=\{\mathrm{MP}, \mathrm{OP}, \mathrm{EP}$, NT\}.

3.2. Methodology. Two dependent variables are used as proxies representing demand: monthly usage intensity and degree of usage imbalance. Two machine-learning models are established in this research. The monthly usage intensity model is used to evaluate the effect of factors influencing usage intensity of exclusive parking space in the analysis spatial units per month. The usage imbalance model is developed to estimate the factors affecting the degree of imbalance of pickup and drop-off at spatial units. The research framework is shown in Figure 3.

3.2.1. Features Selection. Extremely randomized trees algorithm (ET) is used to select the important features to avoid overfitting. The extra-trees algorithm builds an ensemble regression trees. Its two main differences from other treebased ensemble methods, such as random forest [28], are that it divides the nodes by choosing the cut-points completely at random and that it uses the complete learning sample instead of a bootstrap replica to grow the trees. The complete extra-trees algorithm is described in [29]. Relative variance reduction is used to denote the goodness of point splitting. For a sample $S$ and a splitting point $s$ (a feature selected randomly), the goodness of point splitting is expressed as follows:

$$
\begin{aligned}
& G(s, S) \\
& =\frac{\operatorname{var}\{y \mid S\}-\left(\left|S_{l}\right| /|S|\right) \operatorname{var}\left\{y \mid S_{l}\right\}-\left(\left|S_{r}\right| /|S|\right) \operatorname{var}\left\{y \mid S_{r}\right\}}{\operatorname{var}\{y \mid S\}},
\end{aligned}
$$

where $\operatorname{var}\{y \mid S\}$ is the mean squared error of output $y$ in the sample $S$. $S_{l}$ and $S_{r}$ are two subsets of sample $S$. 
TABLE 2: Descriptive statistics of variables.

\begin{tabular}{|c|c|c|c|c|c|c|c|c|}
\hline Variable type & Variable name & Abbr. & Variable type & Mean & Std. & Min. & Med. & Max. \\
\hline \multirow{8}{*}{ Usage intensity } & Workday 06-09 h & I_W0609 & Numerical & 190.13 & 260.16 & 0 & 105 & 2879 \\
\hline & Workday $10-15$ h & I_W1015 & Numerical & 271.26 & 419.04 & 2 & 152 & 5287 \\
\hline & Workday $16-19 \mathrm{~h}$ & I_W1619 & Numerical & 280.09 & 480.29 & 2 & 140 & 6214 \\
\hline & Workday 20-01 h & I_W2001 & Numerical & 285.28 & 430.63 & 0 & 114 & 3987 \\
\hline & Nonworkday 06-09 h & I_NW0609 & Numerical & 61.13 & 86.56 & 0 & 32 & 948 \\
\hline & Nonworkday 10-15 h & I_NW1015 & Numerical & 149.21 & 231.39 & 0 & 80 & 2962 \\
\hline & Nonworkday 16-19 h & I_NW1619 & Numerical & 111.31 & 210.55 & 0 & 54 & 2925 \\
\hline & Nonworkday $20-01 \mathrm{~h}$ & I_NW2001 & Numerical & 110.7 & 173.35 & 0 & 44 & 1860 \\
\hline \multirow{8}{*}{ Usage imbalance } & Workday 06-09 h & IB_W0609 & Numerical & 0.52 & 0.18 & 0.34 & 0.78 & 1 \\
\hline & Workday 10-15 h & IB_W1015 & Numerical & 0.46 & 0.26 & 0.31 & 0.71 & 1 \\
\hline & Workday 16-19 h & IB_W1619 & Numerical & 0.47 & 0.18 & 0.28 & 0.72 & 1 \\
\hline & Workday $20-01 \mathrm{~h}$ & IB_W2001 & Numerical & 0.49 & 0.22 & 0.32 & 0.75 & 1 \\
\hline & Nonworkday 6-09 h & IB_NW0609 & Numerical & 0.48 & 0.23 & 0 & 0.73 & 1 \\
\hline & Nonworkday $10-15$ h & IB_NW1015 & Numerical & 0.41 & 0.20 & 0.19 & 0.65 & 1 \\
\hline & Nonworkday 16-19 h & IB_NW1619 & Numerical & 0.43 & 0.22 & 0.27 & 0.68 & 1 \\
\hline & Nonworkday $20-01 \mathrm{~h}$ & IB_NW2001 & Numerical & 0.45 & 0.27 & 0 & 0.72 & 1 \\
\hline \multirow{4}{*}{ Operational area attributes } & Operational age & $\mathrm{OA}$ & Numerical & 9.82 & 4.01 & 3 & 10 & 44 \\
\hline & Limited-access parking space & LA & Numerical & 1.02 & 3.28 & 0 & 1 & 8 \\
\hline & Underground parking space & UG & Numerical & 0.96 & 2.14 & 0 & 0 & 6 \\
\hline & Exclusive parking space & EPS & Numerical & 4.73 & 1.83 & 0 & 4 & 20 \\
\hline \multirow{5}{*}{ Transportation } & Metro station & MS & Binary & 0.15 & 0.24 & 0 & 0 & 1 \\
\hline & Bus stop & BS & Numerical & 10.28 & 4.96 & 0 & 9 & 44 \\
\hline & Car rental & $\mathrm{CR}$ & Numerical & 0.63 & 0.84 & 0 & 0 & 9 \\
\hline & Intercity coach & IC & Binary & 0.08 & 0.28 & 0 & 0 & 1 \\
\hline & Train station & TS & Binary & 0.06 & 0.31 & 0 & 0 & 1 \\
\hline \multirow{19}{*}{ Built environment } & Residential & RS & Numerical & 35.27 & 42.08 & 0 & 15 & 484 \\
\hline & Public authority & $\mathrm{PA}$ & Numerical & 8.93 & 11.58 & 0 & 2 & 133 \\
\hline & Medical hygiene & $\mathrm{MH}$ & Numerical & 8.47 & 13.37 & 0 & 2 & 235 \\
\hline & Recreation and social & $\mathrm{RC}$ & Numerical & 149.32 & 161.33 & 0 & 53 & 1697 \\
\hline & Culture & $\mathrm{CU}$ & Numerical & 3.21 & 4.11 & 0 & 1 & 53 \\
\hline & Business & $\mathrm{BU}$ & Numerical & 28.6 & 32.69 & 0 & 12 & 471 \\
\hline & University and college & UN & Binary & 0.12 & 0.22 & 0 & 0 & 1 \\
\hline & Industry & IN & Numerical & 3.88 & 2.79 & 0 & 3 & 30 \\
\hline & Public parking & $\mathrm{PP}$ & Numerical & 43.19 & 39.9 & 0 & 20 & 454 \\
\hline & Shopping & $\mathrm{SH}$ & Numerical & 17.67 & 14.16 & 0 & 10 & 126 \\
\hline & Average trip distance & ATD & Numerical & 9.23 & 3.73 & 2.37 & 7.93 & 61.01 \\
\hline & Intersection density & ID & Numerical & 23.86 & 8.45 & 7.22 & 21.98 & 69.47 \\
\hline & POI-mixed entropy & PME & Numerical & 0.78 & 0.1 & 0.193 & 0.82 & 0.98 \\
\hline & Freeway (meter) & FW & Numerical & 486.61 & 2049.96 & 0 & 0 & 15500.97 \\
\hline & Arterial road (meter) & $\mathrm{AR}$ & Numerical & 740.64 & 1356.05 & 0 & 0 & 6557.62 \\
\hline & Secondary road (meter) & SR & Numerical & 620.74 & 1104.11 & 0 & 0 & 6390.57 \\
\hline & Local street (meter) & LS & Numerical & 5611.37 & 3894.72 & 0 & 5760.07 & 16209.97 \\
\hline & One-way road (meter) & OR & Numerical & 3577.73 & 3708.48 & 0 & 2640.8 & 23501.03 \\
\hline & Two-way road (meter) & TR & Numerical & 6657.71 & 3077.18 & 19.84 & 6418.64 & 17526.87 \\
\hline
\end{tabular}




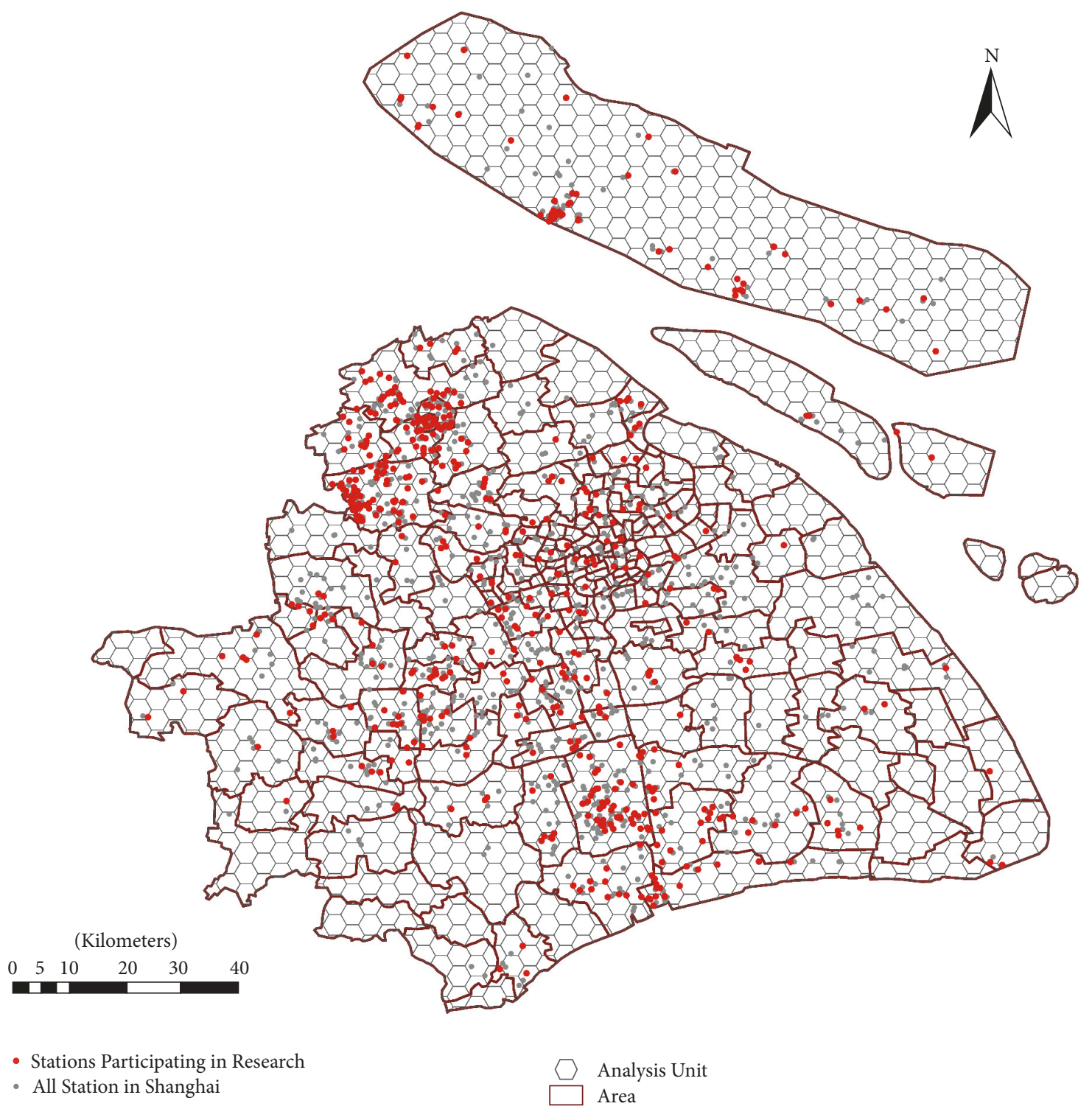

FIGURE 1: Spatial distribution of stations in Shanghai.

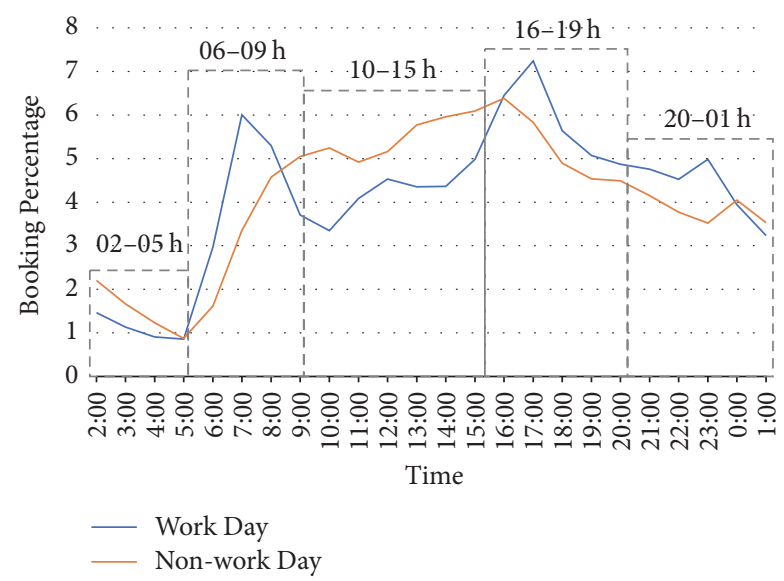

FIgURE 2: EVCARD trip temporal distribution and time division.
Let $G^{i}(s, S)$ be the goodness of $i$ th ET; the feature importance is the average goodness of each ET, which can be expressed as follows:

$$
\mathrm{FI}=\frac{1}{N} \sum_{i=1}^{N} G^{i}(s, S),
$$

where FI denotes the feature importance and $N$ is the number of ET.

Unimportant features have little to no effect on the mean squared error model, while important features should significantly decrease it.

3.2.2. Monthly Usage Intensity Model and Usage Imbalance Model. Considering the unequal duration of time sections, $\tau_{T}$ can be the duration of each $T$, where the unit is hour. 


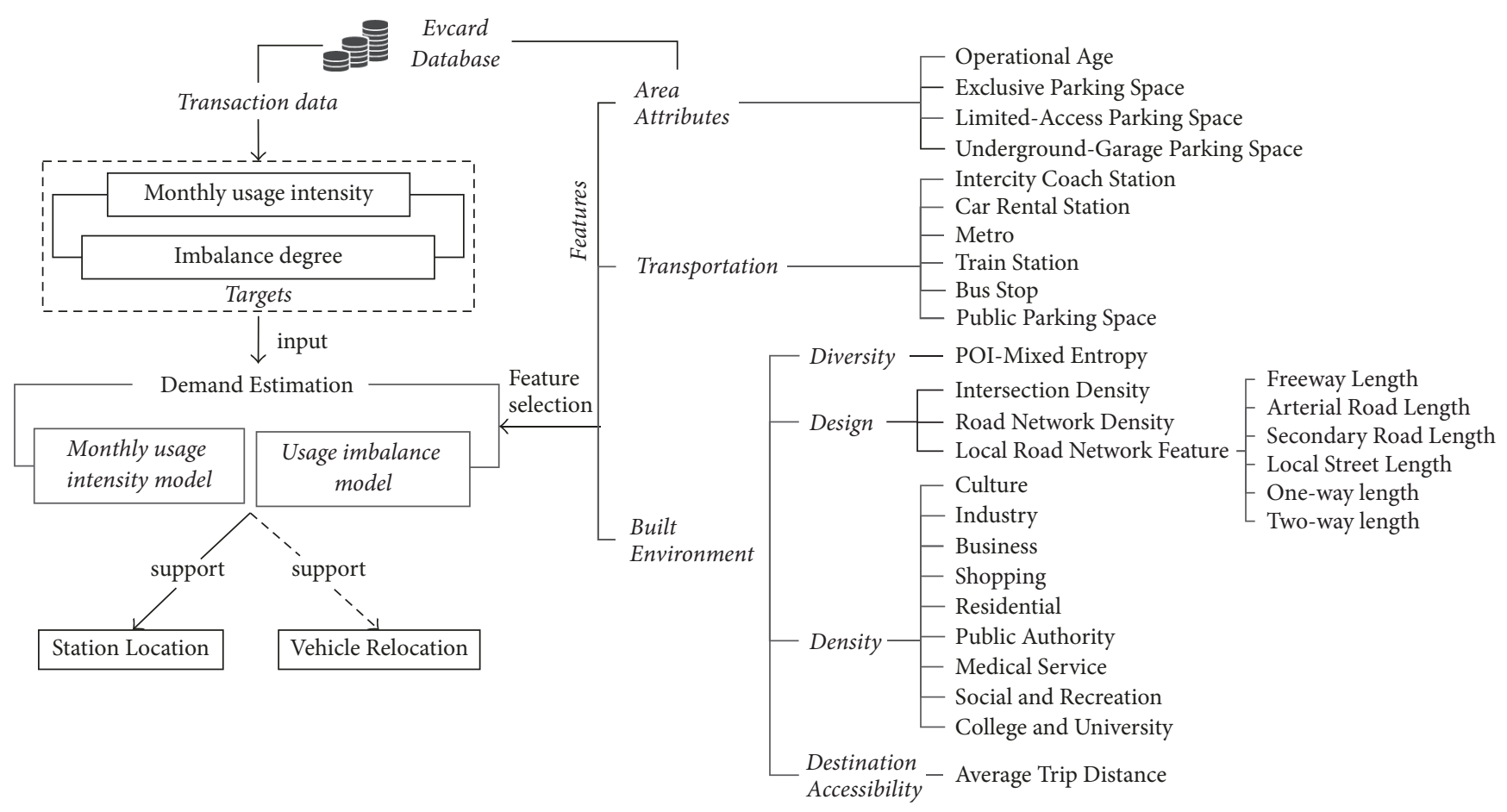

FIGURE 3: Research framework.

Usage intensity is defined as average value per hour of the total amount for pick-up and drop-off in specific time sections of the spatial unit. The calculation formula is shown as follows:

$$
I_{T}=\frac{p_{T}+d_{T}}{l \tau_{T}},
$$

where $I$ represents the usage intensity and $p_{T}$ and $d_{T}$ are the amount of pick-up and drop-off at specific time-interval. $l$ means $l$ months' transaction data is involved in this research; $l$ is 3 here.

We use usage intensity as a proxy of demand rather than the number of bookings because the station is viewed as both origin and destination for the carsharing trip. It not only generates carsharing trip but also attracts it. Therefore, usage intensity is an appropriate index for carsharing demand.

Usage imbalance degree is defined as the ratio of the difference between pick-up and drop-off to the sum of pickup and drop-off in specific time sections of the spatial unit. Half an hour is used as the statistic time interval; then the difference between pick-up and drop-off of each statistic interval is aggregated by corresponding $T$ time section and further divided with the sum of pick-up and drop-off in time section $T$.

Let

$$
P_{T}=\left[\begin{array}{ccc}
p_{11} & \cdots & p_{1 n_{T}} \\
\vdots & \ddots & \vdots \\
p_{m_{T} 1} & \cdots & p_{m_{T} n_{T}}
\end{array}\right],
$$

$$
D_{T}=\left[\begin{array}{ccc}
d_{11} & \cdots & d_{1 n_{T}} \\
\vdots & \ddots & \vdots \\
d_{m_{T} 1} & \cdots & d_{m_{T} n_{T}}
\end{array}\right],
$$

where $P_{T}$ and $D_{T}$ are the pick-up matrix and drop-off matrix in time section $T$ with half-hour statistic interval.

$$
\mathrm{IM}_{T}=\frac{\sum_{i=1}^{m_{T}} \sum_{j=1}^{n_{T}}\left|p_{i j}-d_{i j}\right|}{\sum_{i=1}^{m_{T}} \sum_{j=1}^{n_{T}} p_{i j}+d_{i j}},
$$

where $\mathrm{IM}_{T}$ is the imbalance degree of the spatial unit, $m_{T}$ is the amount of day in time section $T, n_{T}$ is the statistic unit in time section $T$, and $p_{i j}$ and $d_{i j}$ are the pick-up and dropoff quantity in statistic unit of $i$ th day and $j$ th day, respectively.

Given that several types of POIs coexist in the same area with varying degrees, features have multiple collinearity problems that cannot be ignored. Meanwhile, there are many features for samples. Therefore, a linear regression model with $L 1$ and $L 2$ prior as a regularizer, called adaptive elastic net (AEN) regression [30], was developed to predict the usage intensity and the degree of imbalance. The method can be viewed as a combination of elastic net [31] and the adaptive least absolute shrinkage and selection operator (LASSO) [32], which overcome the lack of adaptive LASSO (instability for 
high-dimensional data) and lack of the oracle property for the elastic net. The AEN is defined as follows:

$$
\begin{aligned}
& \widehat{\boldsymbol{\beta}}(\mathrm{AEN})=\left(1+\frac{\lambda_{2}}{n}\right) \\
& \cdot\left\{\underset{\boldsymbol{\beta}}{\operatorname{argmin}}\|y-\mathbf{X} \boldsymbol{\beta}\|_{2}^{2}+\lambda_{2}\|\boldsymbol{\beta}\|_{2}^{2}+\lambda_{1}^{*} \sum_{j=1}^{p} \widehat{w}_{j}\left|\beta_{j}\right|\right\},
\end{aligned}
$$

where

$$
\begin{aligned}
& \widehat{w}_{j}=(|\widehat{\beta}(\mathrm{EN})|)^{-\gamma}, \quad j=1,2, \ldots, p \\
& \widehat{\boldsymbol{\beta}}(\mathrm{EN})=\left(1+\frac{\lambda_{2}}{n}\right) \\
& \quad \cdot\left\{\underset{\boldsymbol{\beta}}{\operatorname{argmin}}\|\mathbf{y}-\mathbf{X} \boldsymbol{\beta}\|_{2}^{2}+\lambda_{2}\|\boldsymbol{\beta}\|_{2}^{2}+\lambda_{1}\|\boldsymbol{\beta}\|_{1}\right\},
\end{aligned}
$$

where $\widehat{\boldsymbol{\beta}}(\mathrm{EN})$ is an elastic net algorithm, $p$ denotes the number of features, $\|\boldsymbol{\beta}\|_{1}=\sum_{j=1}^{p}|\beta|_{1}$ is the $l 1$-norm, and $\|\beta\|_{2}^{2}$ is $l 2$-norm. $\lambda_{1}, \lambda_{2}$, and $\lambda_{1}^{*}$ are weights for the l1-norm, $l 2$ norm, and optimal $\lambda_{1}$, respectively. $\left\{\widehat{w}_{j}\right\}_{j=1}^{p}$ are the adaptive data-driven weights.

\section{Results and Discussion}

A total of 1500 ETs are used to estimate the feature importance so that the ranks and scores of features' importance are stable. We drop the features with importance score not greater than 0.015 . The information of features' importance indicating that the contribution of each feature to prediction is provided in Figures 4 and 5. The features that are not filtered are used for building prediction models. The results of models are shown in Tables 3 and 4.

4.1. Usage Intensity Model. The result shows that $R^{2}$ of intensity models for eight time sections are between 0.404 and 0.583 . The goodness of fit is better than other researches on this issue $[15,23,24]$. Some features show the same direction of influence on usage intensity. However, other factors play a positive or negative effect on the demand depending on the time period. Meanwhile, all factors have different weights for demand across different time periods. This causes the usage intensity to be different across the whole day as shown in Figure 2.

4.1.1. Station-Related Factors. The operational attributes of the spatial unit play an important role in usage intensity across all periods. The longer the first station operates in spatial unit, the greater the intensity is because the service and location of a station are getting familiarized by users gradually. Limited-access parking space has constantly provided negative effect on usage intensity. Exclusive parking space represents the supply level partly. It positively affects usage intensity. However, these factors are endogenous items, and the carsharing operator can improve these factors as possible as it can. The more important factors are exogenous variables, such as the built environment and transportation in the spatial unit.

4.1.2. Built Environment Factors. Built environment factors show the diverse effect on usage intensity in different time periods. College and university constantly has positive influence. In contrast, the industrial area shows negative effect. Residential, culture, public authority, and medical hygiene area impose negative influence on usage intensity in special time period. Other factors have opposite effect depending on the time period. Recreation area has a negative influence on usage intensity in workday's early peak and positive influence during nonworking time sections. More POI-related shopping is within the spatial unit, and usage intensity shows more increase in evening peak and night.

POI-mixed entropy plays a positive role in the usage intensity during working day at around 16:00-2:00. The average trip distance is a special factor. It has positive effect on the evening peak of working days and night of nonworking days, which implies that users might tend to use carsharing for further distance trip during these periods. Additionally, it has negative effect during 6:00-10:00 in nonworking days, which indicates that short distance trip of carsharing tends to be at 6:00-10:00 in nonworking days.

Areas with higher intersection density, mainly during nonworking time sections, improve usage intensity because of good accessibility. It is unexpected that the length of the local street and one-way road generally has negative effect and two-way road has a positive effect on usage intensity. Given that the local street and one-way street are more walkfriendly, the result is opposite to some research. This finding may be attributed to our use of intensity which includes pick-up and drop-off instead of pick-up only to count as an indicator of demand. Many local streets and one-way streets would make it more difficult for users to find parking space. In contrast, more two-way roads but less one-way and local streets means simpler road network.

4.1.3. Transportation Factors. Considering the transportation factors, unexpectedly, traditional car rental station has a positive effect on station usage intensity at 6:00-16:00 on working days and 10:00-20:00 on nonworking days. Despite being shown in literature that car rental and carsharing have competitive relationship in medium distance trip [1], this result indicates a more complicated relationship between carsharing and car rental. Moreover, intercity coach station has key role and positive relation during 6:00-16:00 on nonworking days, which has not been reported in any current literature. Intercity coach stations are generally far from the center of the city and passengers have no personal car while taking some packages, which could be the main reason for the demand in using carsharing to connect with intercity coach.

For public transportation, the existence of metro station negatively affects carsharing station usage intensity during morning peak and evening peak on working days. This finding could be attributed to the belief that the metro is more reliable for commuting compared to ground traffic, and commute to work is time-limited. Meanwhile, the metro imposes positive effect during nonworkday, which implies 

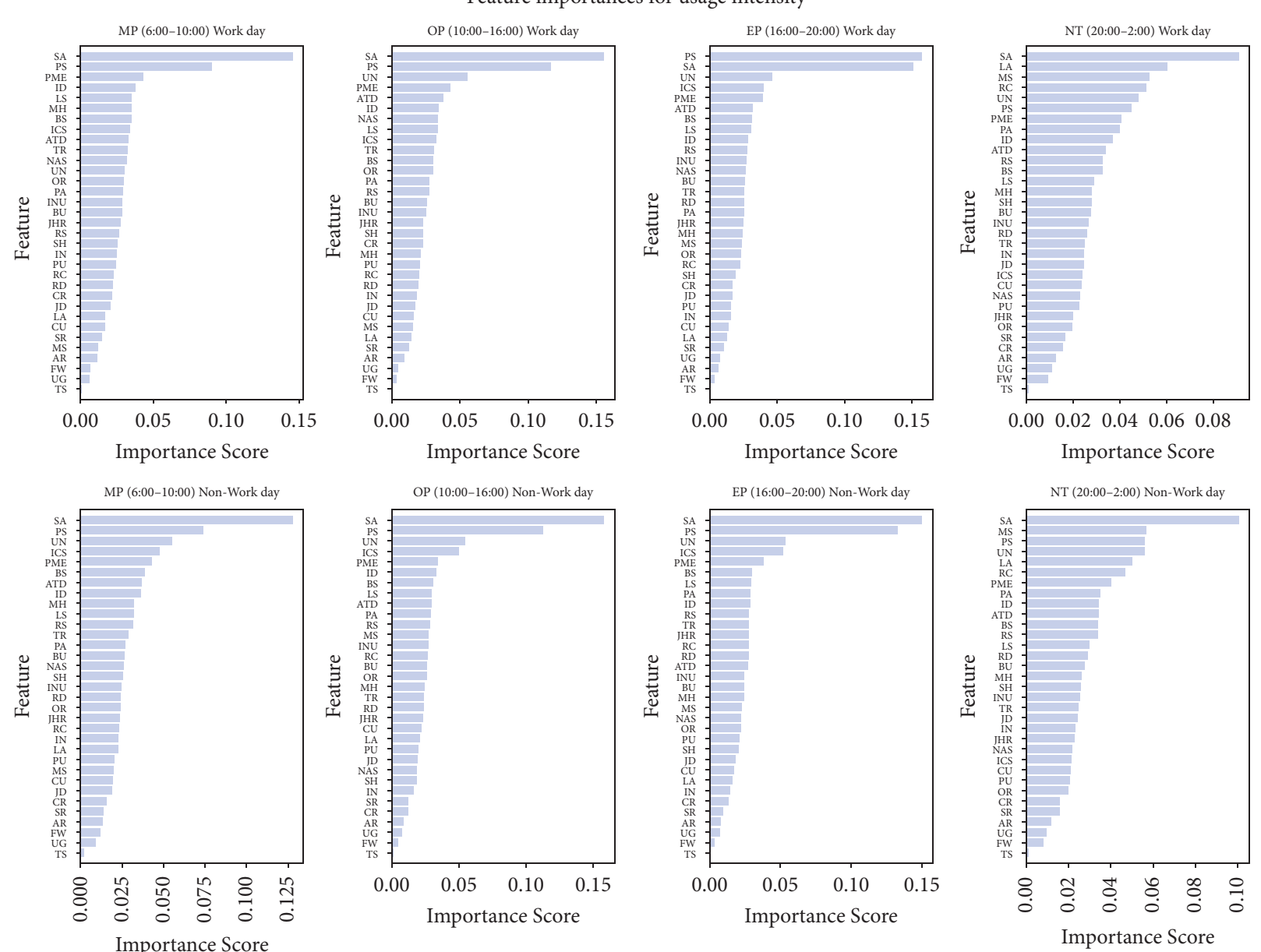

FIgURE 4: Feature importance for usage intensity.

that carsharing users are using the metro to connect carsharing during nonworkday. The metro competes with carsharing in rush hours but they cooperate with each other during nonworkdays. This relationship shows a policy potential for the government to promote diversified mobility without deteriorating ground traffic condition.

The bus stop has a positive effect during 6:00-20:00 in both workday and nonworkday, which is similar to literature [20]. This could be attributed to the good accessibility of the area near bus stops. Therefore, the exposed rate of carsharing will be high if the station is placed in nearby bus stop. This explains the nonsignificance during 10:00-20:00 in nonworkdays because the main purpose of nonworkdays is leisure, which requires higher sensitivity to comfort and lower sensitivity to price.

Public parking space has a significantly negative impact on usage intensity. The result implies that more public parking spaces result in more private vehicle trips rather than carsharing. Since private vehicle is very inefficient in using parking space, if a part of the public parking space is replaced with carsharing exclusive parking space gradually, it can (1) save huge area of high-value land in the center of the city and (2) reduce private vehicle usage.
4.2. Usage Imbalance Model. $R^{2}$ of usage imbalance models for eight time sections are between 0.217 and 0.514 , which are worse than the usage intensity models. The worst imbalance model is that of the morning peak in a nonworkday. The less usage in the early morning of the nonworkday results in a few factors showing significant effect.

4.2.1. Station-Related Factors. With increasing operation age, the degree of imbalance decreases for all time periods. A spatial unit with more limited-access parking space means that it only serves lower proportion of users, and the demand diversity (purpose, departure time, and arriving time) within the spatial unit is lower. The same reason results in the similar appearance effect of underground-garage parking spaces. Therefore, the operator should locate less parking space on limited access and underground garage.

4.2.2. Built Environment Factors. At the built environment factor, residential, public authority, business, and industrial area continually play a negative role to increase the degree of imbalance in special time period. Recreation, medical hygiene, university, and shopping area have different effect in different time periods. Among them, the university has a 

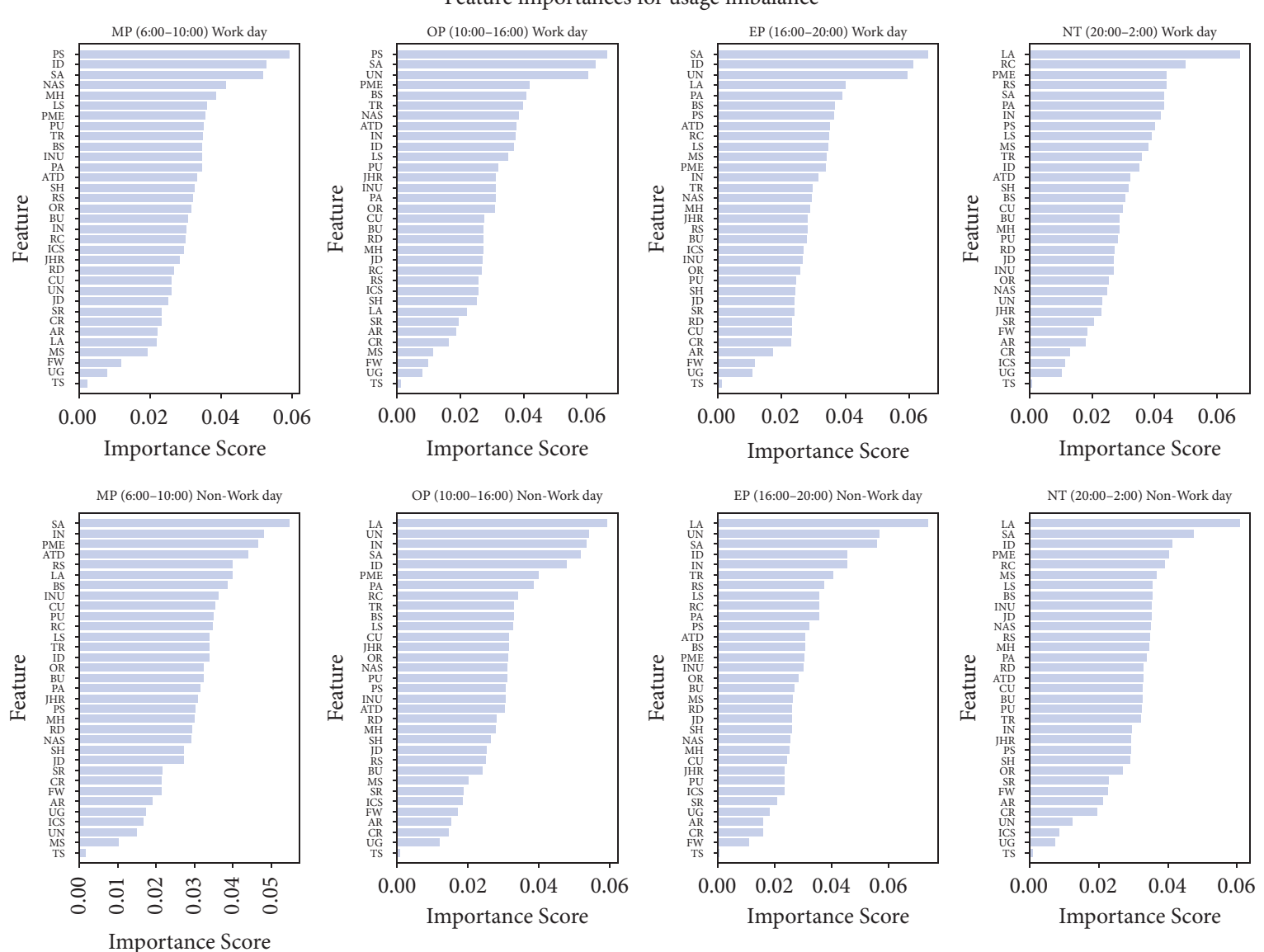

FIGURE 5: Feature importance for usage imbalance.

positive effect on station balance in most of the time sections, given that many adult students live in this area. These people have less time constraint, flexible travel time, and diverse travel purpose. However, it appears as a negative effect during 6:00-10:00 on nonworkday, which could mean that people living in these areas tend to go out of campus during this time section.

Intersection density represents accessibility partially. It can influence people who are unfamiliar with station location to access the station. More people using the station can generate and attract diversified and compensative usage of pick-up and drop-off. Our new finding is that a longer arterial road and secondary road lead to higher degree of usage imbalance in the spatial unit. By contrast, the local street results in more balanced carsharing spatial unit. Analogously, more two-way roads show higher degree of imbalance, and more one-way streets result in lower imbalance. This could be attributed to the increased possibility of imbalance in a specified area because of higher usage intensity.

POI-mixed entropy reduces the degree of imbalance during morning peak and night of workdays and nonworkday nights. The diversified demand can reduce the usage imbalance. However, it increases the degree of station imbalance during 10:00-16:00 on working days, which could be attributed to the low usage intensity of the high diversity areas in this time section indicative of scattered pick-up and drop-off. It causes the imbalance of the demand for drop-off and pick-up during the statistical interval (half hour).

4.2.3. Transportation Variables. For transportation variables, metro stations play a significantly positive role in station usage balance because of the huge crowd nearby metro, and the trip purpose and time are diverse. On the other hand, it is implied that carsharing has a closer relationship with metro, and there might be a demand for connection between carsharing and metro. Therefore, it is implied that those two modes can compensate each other. By contrast, the bus stop shows opposite effect (negative) on usage imbalance. Given that the main difference between the bus and the metro is that the former runs on the ground where the uncertainty of trip duration is larger, the significant finding implies that carsharing attracts a part of the bus passengers unidirectionally, even in early peak and evening peak on workday. Therefore, from the government viewpoint, carsharing station should not be located near a bus stop, which results in a transit trip transferring to a car trip. Besides, car rental station appears to have positive effect on station usage balance in partial time section. It is implied that there is a demand of using carsharing to connect with car rental.

The results of imbalance model are shown in Table 4. 
TABLE 3: The result of monthly usage intensity model.

\begin{tabular}{|c|c|c|c|c|c|c|c|c|}
\hline \multirow{2}{*}{ Features } & \multicolumn{4}{|c|}{ Workday } & \multicolumn{4}{|c|}{ Nonworkday } \\
\hline & $06-09 \mathrm{~h}$ & $10-15 \mathrm{~h}$ & $16-19 \mathrm{~h}$ & $20-01 \mathrm{~h}$ & $06-09 \mathrm{~h}$ & $10-15 \mathrm{~h}$ & $16-19 \mathrm{~h}$ & $20-01 \mathrm{~h}$ \\
\hline \multicolumn{9}{|c|}{ Area attributes } \\
\hline $\mathrm{OA}$ & 0.790 & 0.664 & 0.628 & 0.679 & 0.748 & 0.652 & 0.598 & 0.597 \\
\hline LA & -0.073 & -0.040 & -0.123 & -0.387 & -0.178 & -0.183 & -0.163 & -0.323 \\
\hline EPS & 0.506 & 0.645 & 0.560 & 0.348 & 0.293 & 0.427 & 0.435 & 0.265 \\
\hline \multicolumn{9}{|c|}{ Built environment } \\
\hline \multicolumn{9}{|c|}{ Density } \\
\hline RS & -0.081 & & & & -0.052 & & -0.030 & \\
\hline $\mathrm{PA}$ & -0.016 & & -0.024 & -0.448 & -0.171 & -0.067 & -0.164 & -0.283 \\
\hline $\mathrm{RC}$ & -0.055 & & & 0.318 & & 0.017 & 0.151 & 0.019 \\
\hline $\mathrm{CU}$ & -0.149 & & -0.032 & -0.153 & -0.053 & & & -0.026 \\
\hline MH & & & & -0.059 & & & -0.093 & -0.071 \\
\hline $\mathrm{BU}$ & -0.047 & & 0.012 & & -0.193 & -0.017 & -0.054 & \\
\hline UN & 0.154 & 0.209 & 0.175 & 0.220 & 0.228 & 0.213 & 0.209 & 0.233 \\
\hline IN & -0.211 & -0.104 & -0.150 & -0.192 & -0.185 & -0.148 & -0.155 & -0.061 \\
\hline SH & -0.087 & -0.025 & -0.017 & 0.077 & -0.040 & & 0.033 & 0.038 \\
\hline \multicolumn{9}{|l|}{ Design } \\
\hline ID & & 0.103 & 0.173 & 0.484 & 0.307 & 0.301 & 0.356 & 0.413 \\
\hline LS & -0.050 & & & 0.071 & -0.056 & & -0.045 & \\
\hline OR & -0.004 & & & -0.099 & -0.045 & -0.063 & -0.087 & -0.076 \\
\hline $\mathrm{TR}$ & & & & 0.013 & & & & 0.026 \\
\hline \multicolumn{9}{|l|}{ Diversity } \\
\hline PME & & -0.020 & & 0.336 & 0.059 & & 0.042 & 0.300 \\
\hline \multicolumn{9}{|c|}{ Destination accessibility } \\
\hline ATD & & 0.186 & 0.236 & & -0.179 & & & 0.131 \\
\hline \multicolumn{9}{|c|}{ Transportation } \\
\hline MS & -0.163 & & -0.192 & 0.188 & 0.094 & 0.136 & 0.107 & 0.187 \\
\hline BS & 0.112 & 0.162 & 0.210 & & 0.239 & 0.136 & 0.101 & \\
\hline $\mathrm{CR}$ & 0.164 & 0.171 & 0.064 & & & 0.012 & 0.029 & \\
\hline ICS & 0.186 & 0.211 & 0.189 & 0.315 & 0.327 & 0.288 & 0.306 & 0.270 \\
\hline $\mathrm{PP}$ & -0.218 & & -0.184 & -0.166 & & & -0.229 & -0.175 \\
\hline$R 2$ & 0.422 & 0.404 & 0.412 & 0.583 & 0.511 & 0.488 & 0.504 & 0.539 \\
\hline
\end{tabular}

Combining these two models, the significant features can be arranged as shown in Figure 6. The features within the range of the dotted lines and located on $x$-axis or $y$-axis only have significant impact on single dependent variables. Meanwhile, the others in the outer side beyond the dotted range are significant on both dependent variables.

Given an average value of area attributes as shown in Table 2, we get some appropriate location of carsharing station based on the result of usage intensity model and usage imbalance model, respectively. We divide location of Shanghai into three levels in proportion as $25 \%, 50 \%$, and $25 \%$, firstly. Then combining results of two models, the location can be divided into five levels, prior, recommended, medium, not recommended, avoid, as Figure 7 shows. We find that central area takes a relatively large proportion of prior level area to locating. Given that a lot of central areas of city are appropriate to locating carsharing station and carsharing is more efficient in using parking space, we suggest that more carsharing exclusive parking space can be used to replace public parking space to decrease usage of private vehicle and save parking space. Moreover, many suburb locations are evaluated as prior or recommended level by models. This means that the usage scenarios of carsharing are wider than central area. If these suburb areas can be developed adequately, the usage scenarios related to outskirts will take on more trips.

\section{Conclusions}

This study focused on the largest station-based OWC program in Shanghai, China. There are many approaches to estimate carsharing demand according to research objects. 
TABLE 4: The results of imbalance model.

\begin{tabular}{|c|c|c|c|c|c|c|c|c|}
\hline \multirow{2}{*}{ Features } & \multicolumn{4}{|c|}{ Workday } & \multicolumn{4}{|c|}{ Nonworkday } \\
\hline & $06-09 \mathrm{~h}$ & $10-15 \mathrm{~h}$ & $16-19 \mathrm{~h}$ & $20-01 \mathrm{~h}$ & 06-09h & $10-15 \mathrm{~h}$ & $16-19 \mathrm{~h}$ & $20-01 \mathrm{~h}$ \\
\hline \multicolumn{9}{|c|}{ Area attributes } \\
\hline OA & -0.189 & -0.146 & -0.189 & -0.003 & -0.044 & -0.102 & -0.117 & -0.012 \\
\hline LA & 0.011 & & 0.079 & 0.075 & & 0.080 & 0.100 & 0.002 \\
\hline UG & & & & 0.147 & & & 0.020 & \\
\hline \multicolumn{9}{|c|}{ Built environment } \\
\hline \multicolumn{9}{|c|}{ Density } \\
\hline RS & -0.120 & & -0.015 & & -0.210 & & & \\
\hline $\mathrm{PA}$ & 0.028 & & 0.083 & & & 0.021 & 0.025 & \\
\hline $\mathrm{RC}$ & & & & 0.125 & & -0.051 & -0.067 & 0.173 \\
\hline $\mathrm{MH}$ & -0.028 & & 0.136 & & & & & \\
\hline $\mathrm{BU}$ & & & 0.104 & & & 0.116 & 0.077 & \\
\hline UN & -0.064 & -0.101 & -0.101 & -0.052 & 0.041 & -0.076 & -0.092 & \\
\hline IN & 0.044 & 0.095 & 0.082 & & & 0.024 & 0.014 & \\
\hline SH & -0.010 & 0.022 & 0.047 & & & -0.011 & 0.045 & \\
\hline \multicolumn{9}{|l|}{ Design } \\
\hline ID & -0.204 & -0.088 & -0.174 & & & -0.097 & -0.119 & -0.099 \\
\hline $\mathrm{AR}$ & 0.113 & & 0.103 & & & 0.018 & 0.026 & \\
\hline SR & 0.351 & & 0.265 & & & 0.107 & & \\
\hline LS & -0.071 & & -0.054 & -0.056 & & 0.014 & -0.049 & \\
\hline OR & & & -0.058 & & & -0.001 & -0.050 & \\
\hline TR & & 0.034 & 0.085 & & & & 0.061 & \\
\hline \multicolumn{9}{|l|}{ Diversity } \\
\hline PME & -0.048 & 0.058 & 0.024 & -0.139 & & 0.052 & 0.004 & -0.059 \\
\hline \multicolumn{9}{|c|}{ Destination accessibility } \\
\hline ATD & -0.071 & -0.133 & -0.107 & & & -0.046 & -0.125 & \\
\hline \multicolumn{9}{|c|}{ Transportation } \\
\hline MS & -0.181 & & -0.046 & -0.060 & & -0.046 & -0.060 & -0.096 \\
\hline BS & 0.119 & 0.049 & 0.151 & & 0.014 & 0.013 & & \\
\hline $\mathrm{CR}$ & -0.030 & -0.051 & -0.166 & & 0.016 & & & \\
\hline $\mathrm{PP}$ & -0.158 & & -0.127 & & & & & \\
\hline$R 2$ & 0.424 & 0.394 & 0.514 & 0.329 & 0.217 & 0.419 & 0.447 & 0.315 \\
\hline
\end{tabular}

However, the station-based one-way system is rarely investigated. Meanwhile, many research investigations focus on the usage rate, vehicle hour traveled (VHT), and many others, but the station usage imbalance has not yet been investigated. This study addressed this gap.

In this study, multiple linear regression models and beta regression model are developed to analyze how different factors affect station usage intensity and degree of station imbalance across different periods. The conclusions are summarized as follows:

(1) The attributes of spatial unit constantly appear to have significant effect on the demand characteristics. However, many built environment and transportation factors have a different effect on the demand in different time periods. This is the main reason why carsharing demand appears to be dynamic across time periods.
(2) For usage intensity, the university, high POI-mixed entropy, high intersection density area, and area including a metro station, bus stop, car rental station, and intercity coach have positive influence on usage intensity. However, industrial, residential, culture, public authority, and medical hygiene areas show negative effect in different time periods, in which layout should be avoided by carsharing stations.

(3) For the degree of usage imbalance, it will decrease along with the increase in operation age. Limitedaccess parking space enhances usage imbalance. Residential, public authority, business, and industrial areas continually play a negative role to increase the degree of imbalance in special time period. The area with the university, high intersection density, high POI-mixed entropy, and more local streets and oneway roads lead to more balanced operational area. 


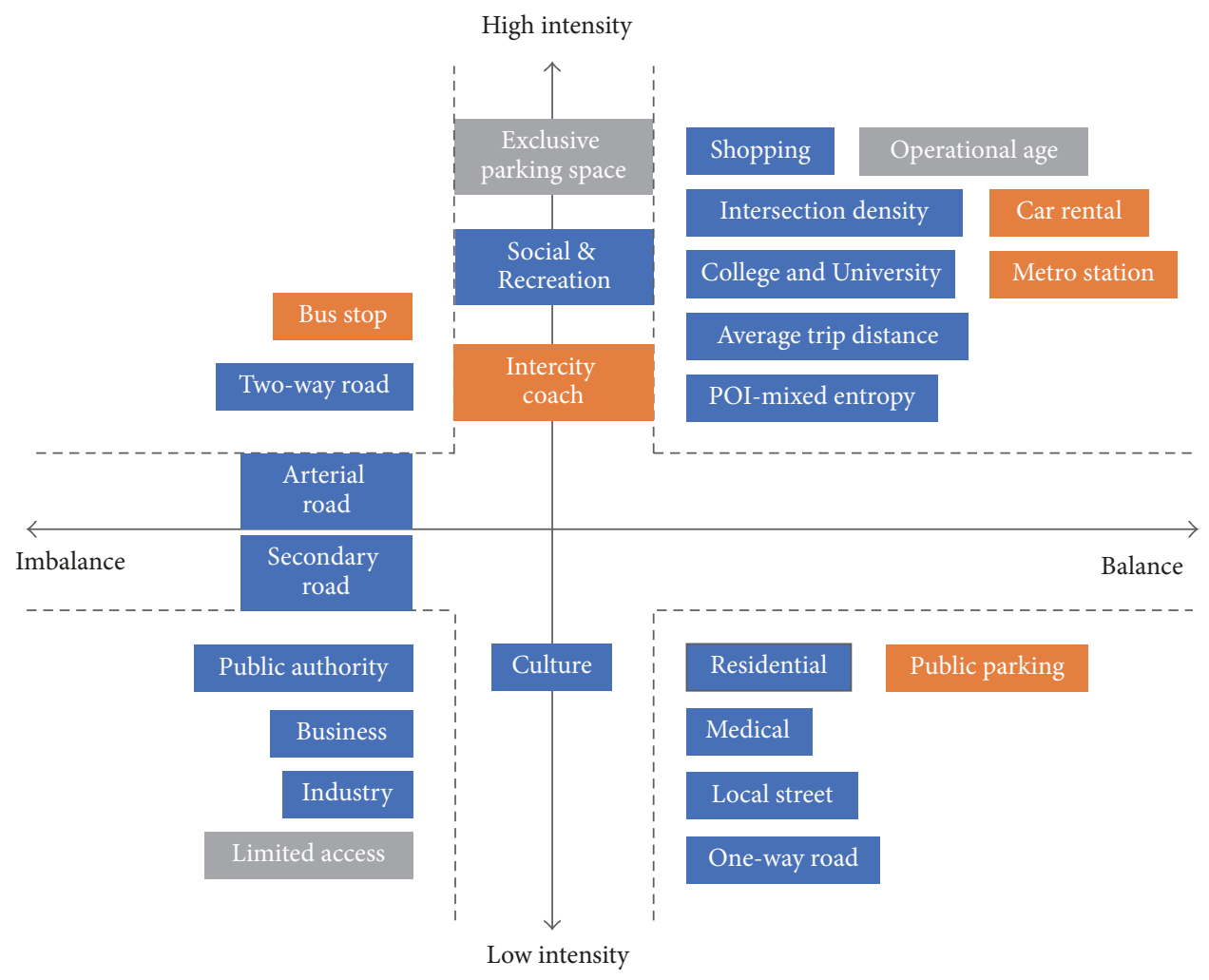

Area attributes

Built Environment

Transportation

FIGURE 6: Influence diagram of statistically significant independent variables.

(4) Areas with adequate public parking space will attract more personal vehicle use rather than carsharing trip. Given that carsharing is more efficient in using parking space, we suggest that public parking spaces should be gradually converted to carsharing exclusive parking space. This will increase the usage efficiency of the limited number of parking spaces and reduce personal vehicle usage while having a flexible car trip still available.

(5) For public transportation, the metro and bus are significantly different for carsharing. The metro has a strong advantage over carsharing in the morning and evening peak on workdays because of its certainty of trip duration. Thus, carsharing cannot attract passengers from the metro in rush hour. Meanwhile, they appear to connect with each other in another time period, which is a complementary relationship. However, the bus is similar to carsharing, which runs on the ground but lacks the comfort and personality of carsharing. Thus, carsharing has a related advantage over the bus, which results in some bus passengers transferring to carsharing unidirectionally. Therefore, we suggest that the government should encourage carsharing station layout near a metro station but not a bus stop.
Usage intensity is related to profits, and the degree of station imbalance is related to dispatching cost. From the carsharing operator viewpoint, the purpose of the carsharing station is to minimize the cost to obtain the maximum benefit. Thus, the results shown in Figure 6 can be viewed as a guidance of carsharing station layout for maximizing benefit. The features in the first quadrant lead to higher usage intensity and lower imbalance degree; meanwhile, features in the third quadrant result in lower usage intensity and higher imbalance degree. Therefore, carsharing station should be given priority to locating at area with features in the first quadrant and setting up stations in areas with features in the third quadrant should be avoided. Other factors can be selected as secondary, such as stations nearby metro stations, which only decrease station usage intensity during peak time section on workdays. However, it might be a good choice to select the station near other stations so that the imbalance level can be dramatically decreased during most of the time sections.

The method of modeling for different time sections reveals, to a certain extent, the temporal dynamics patterns of the demand, which can provide guidance for vehicle relocation. In college and university areas, the imbalance level is high at 6:00-9:00 on nonworking days, which shows that extra dispatch is needed during this time section. However, the research conclusion is built upon long-term measurement 

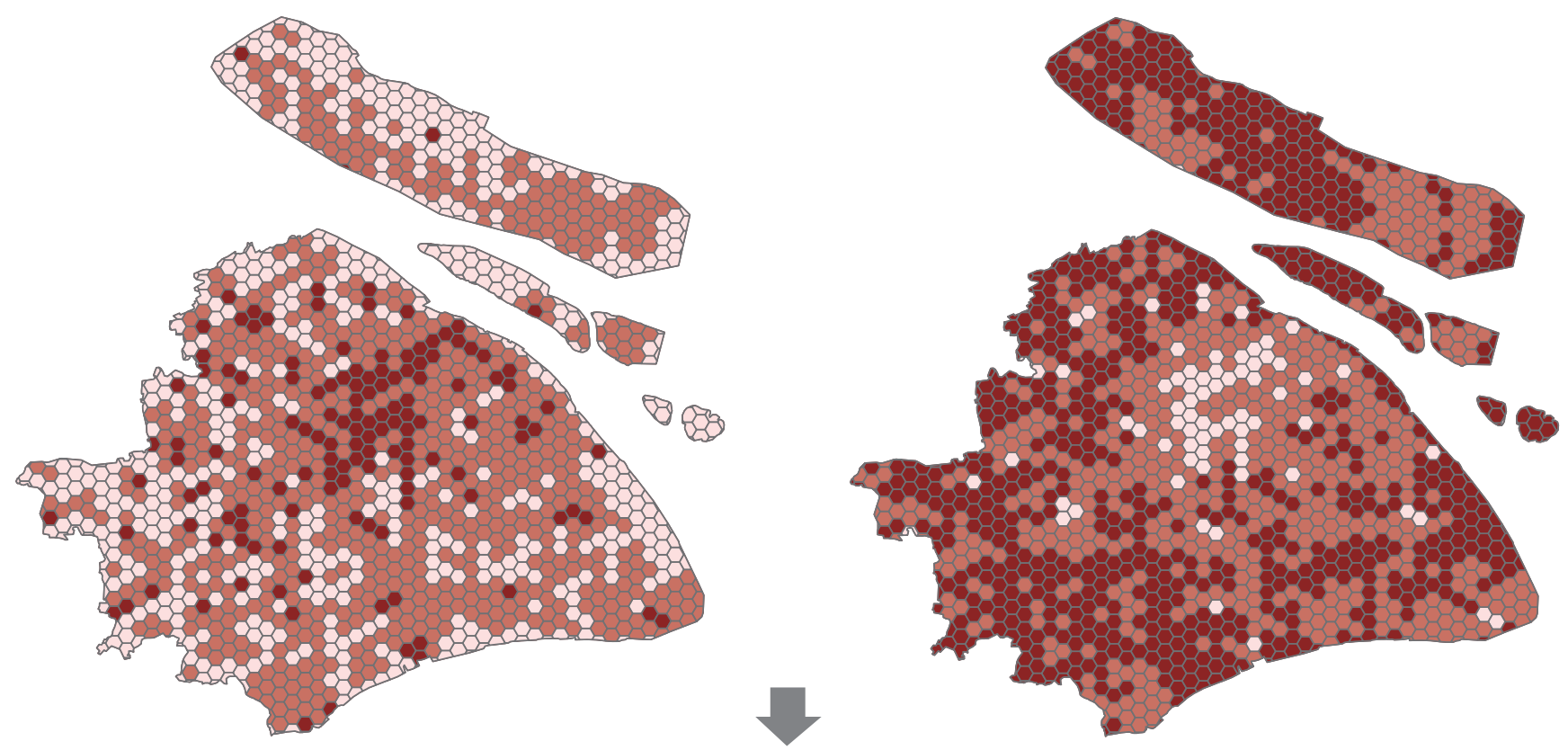

Intensity

$\square 0-1320$

Imbalance

1320-1886

1886-3780
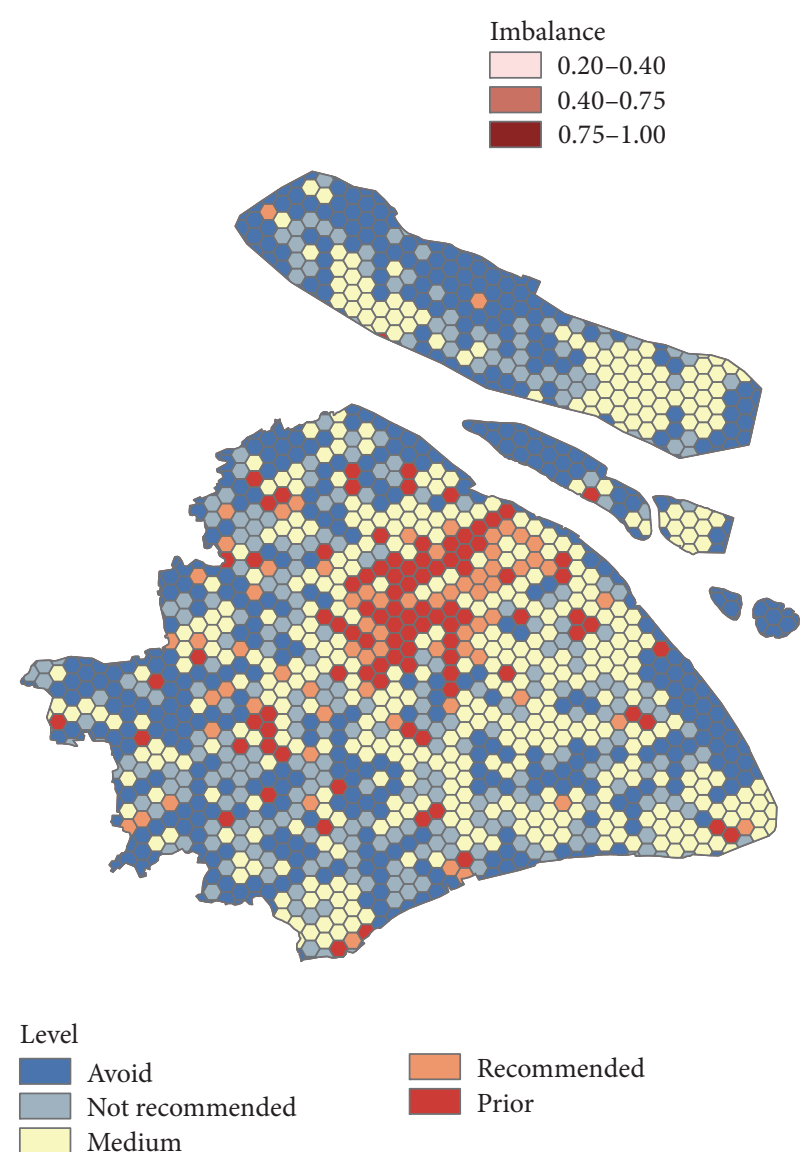

FIGURE 7: Combining usage intensity model and imbalance model to locating carsharing station in Shanghai. 
(three months). Thus, it can provide a noninstant dispatch strategy. We believe that it is strategically advantageous to arrange vehicle in advance based on demand dynamics pattern concluded by this research. Then, an instant dispatch method is used for adjustment accordingly.

There are three main limitations in this research:

(1) The statistics radium station is $800 \mathrm{~m}$, and it only refers to the value in the research of public transit. Although the range of $800 \mathrm{~m}$ is widely used in carsharing areas [24], the service range of carsharing stations in different zones and different traffic conditions can vary.

(2) The categorization of time section is only based on the time distribution feature of bookings, but more reasonable time categorization shall be an improvement direction.

(3) In the calculation of station imbalance level, statistic time interval is very important. Too small interval might cause high imbalance level, while too big interval may cause low level of imbalance. We infer that statistic time interval should depend on different usage intensities in each spatial unit, but this limitation will be improved in future research.

\section{Conflicts of Interest}

The authors declare that they have no conflicts of interest regarding the publication of this paper.

\section{Acknowledgments}

The authors would like to acknowledge the Shanghai International Automobile City Co., Ltd. and Global Carsharing \& Rental Co., Ltd. for providing the precious data of EVCARD in this research. This study is supported by the National Natural Science Foundation of China (71734004), China National Key Technology R\&D Program (2015BAG11B01), and Open Research Funding of "Gaofeng” Discipline (2016J012307).

\section{References}

[1] A. Millard-Ball, "Where and how it succeeds," Transportation Research Board, 2005.

[2] E. Martin, S. Shaheen, and J. Lidicker, "Impact of carsharing on household vehicle holdings: Results from North American shared-use vehicle survey," Transportation Research Record: Journal of the Transportation Research Board, vol. 2143, pp. 150158, 2010.

[3] J. T. Schure, F. Napolitan, and R. Hutchinson, "Cumulative impacts of carsharing and unbundled parking on vehicle ownership and mode choice," Transportation Research Record, no. 2319, pp. 96-104, 2012.

[4] S. A. Shaheen, C. Rodier, and G. Murray, Carsharing and Public Parking Policies: Assessing Benefits, Costs, and Best Practices in North America, 2010.

[5] E. W. Martin and S. A. Shaheen, "Greenhouse gas emission impacts of carsharing in North America," IEEE Transactions on
Intelligent Transportation Systems, vol. 12, no. 4, pp. 1074-1086, 2011.

[6] H. Nijland, J. Van Meerkerk, and A. Hoen, Impact of Car Sharing on Mobility and $\mathrm{CO}_{2}$ Emissions, PBL Note, 2015.

[7] A. Bieszczat and J. Schwieterman, Are Taxes on Carsharing Too High? A Review of the Public Benefits and Tax Burden of an Expanding Transportation Sector, Chaddick Institute for Metropolitan Development, DePaul University, 2011.

[8] J. Firnkorn and M. Müller, "Free-floating electric carsharingfleets in smart cities: The dawning of a post-private car era in urban environments?" Environmental Science \& Policy, vol. 45, pp. 30-40, 2015.

[9] G. D. Kim, J. Park, and J. D. Woo, Investigating the Characteristics of Carsharing Usage Pattern for Public Rental Housing Complexes: A Case Study in South Korea, 2017.

[10] F. Ferrero, G. Perboli, and A. Vesco, Car-Sharing Services-Part a Taxonomy and Annotated Review, Montréal, Canada, 2015.

[11] R. Katzev, "Car Sharing: A New Approach to Urban Transportation Problems," Analyses of Social Issues and Public Policy, vol. 3, no. 1, pp. 65-86, 2003.

[12] C. Costain, C. Ardron, and K. N. Habib, "Synopsis of users' behaviour of a carsharing program: A case study in Toronto," Transportation Research Part A: Policy and Practice, vol. 46, no. 3, pp. 421-434, 2012.

[13] K. M. N. Habib, C. Morency, M. T. Islam, and V. Grasset, "Modelling users' behaviour of a carsharing program: Application of a joint hazard and zero inflated dynamic ordered probability model," Transportation Research Part A: Policy and Practice, vol. 46, no. 2, pp. 241-254, 2012.

[14] A. De Lorimier and A. M. El-Geneidy, "Understanding the factors affecting vehicle usage and availability in carsharing networks: a case study of communauto carsharing system from Montréal, Canada," International Journal of Sustainable Transportation, vol. 7, no. 1, pp. 35-51, 2012.

[15] K. Kim, "Can carsharing meet the mobility needs for the low-income neighborhoods? Lessons from carsharing usage patterns in New York City," Transportation Research Part A: Policy and Practice, vol. 77, pp. 249-260, 2015.

[16] J. Kang, K. Hwang, and S. Park, "Finding factors that influence carsharing usage: Case study in seoul," Sustainability, vol. 8, no. 8, p. 709, 2016.

[17] R. Seign and K. Bogenberger, "Model-Based Design of FreeFloating Carsharing Systems," in Proceedings of the Transportation Research Board 94th Annual Meeting, 2015.

[18] M. Khan and R. Machemehl, The Impact of Land-Use Variables on Free-Floating Carsharing Vehicle Rental Choice and Parking Duration, Seeing Cities Through Big Data, Springer International Publishing, 2017.

[19] S. Schmöller and K. Bogenberger, "Analyzing External Factors on the Spatial and Temporal Demand of Car Sharing Systems," Procedia - Social and Behavioral Sciences, vol. 111, pp. 8-17, 2014.

[20] S. Wagner, T. Brandt, and D. Neumann, "In free float: Developing Business Analytics support for carsharing providers," OMEGA - The International Journal of Management Science, vol. 59, pp. 4-14, 2016.

[21] K. Klemmer, S. Wagner, C. Willing, and T. Brandt, Explaining Spatio-Temporal Dynamics in Carsharing: A Case Study of Amsterdam, 2016.

[22] S. Schmöller, S. Weikl, J. Müller, and K. Bogenberger, "Empirical analysis of free-floating carsharing usage: The munich and berlin case," Transportation Research Part C: Emerging Technologies, vol. 56, pp. 34-51, 2015. 
[23] T. Stillwater, P. L. Mokhtarian, and S. A. Shaheen, "Carsharing and the built environment: Geographic information system based study of one U.S. operator," Transportation Research Record: Journal of the Transportation Research Board, vol. 2110, pp. 27-34, 2009.

[24] C. Celsor and A. Millard-Ball, "Where does carsharing work? Using geographic information systems to assess market potential," Transportation Research Record: Journal of the Transportation Research Board, vol. 1992, pp. 61-69, 2007.

[25] Y. Jiang, P. Gu, F. Chen et al., Measuring Transit-Oriented Development in Quantity and Quality: A Case of 24 Cities with Urban Rail Systems in China, 2017.

[26] R. Cervero and K. Kockelman, "Travel demand and the 3Ds: density, diversity, and design," Transportation Research Part D: Transport and Environment, vol. 2, no. 3, pp. 199-219, 1997.

[27] R. Ewing and R. Cervero, "Travel and the built environment: a meta-analysis," Journal of the American Planning Association, vol. 76, no. 3, pp. 265-294, 2010.

[28] L. Breiman, "Random forests," Machine Learning, vol. 45, no. 1, pp. 5-32, 2001.

[29] P. Geurts, D. Ernst, and L. Wehenkel, "Extremely randomized trees," Machine Learning, vol. 63, no. 1, pp. 3-42, 2006.

[30] H. Zou and H. H. Zhang, "On the adaptive elastic-net with a diverging number of parameters," Annals of Statistics, vol. 37, no. 4, pp. 1733-1751, 2009.

[31] H. Zou and T. Hastie, "Regularization and variable selection via the elastic net," Journal of the Royal Statistical Society, vol. 67, no. 2, pp. 768-768, 2005.

[32] H. Zou, "The Adaptive Lasso and Its Oracle Properties," Publications of the American Statistical Association, vol. 101, no. 476, pp. 1418-1429, 2006. 


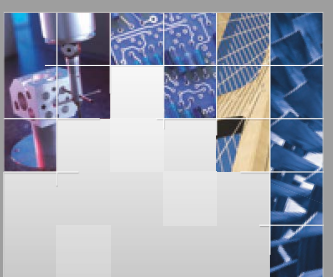

\section{Enfincering}
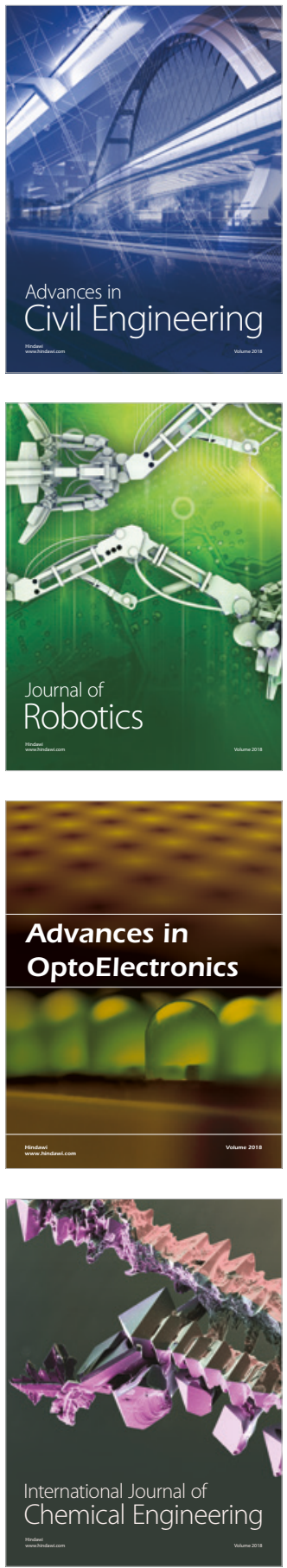

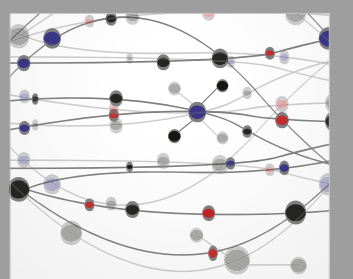

\section{Rotating \\ Machinery}

The Scientific World Journal

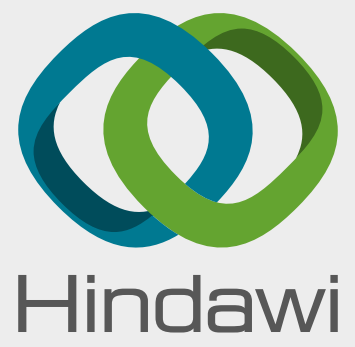

Submit your manuscripts at

www.hindawi.com
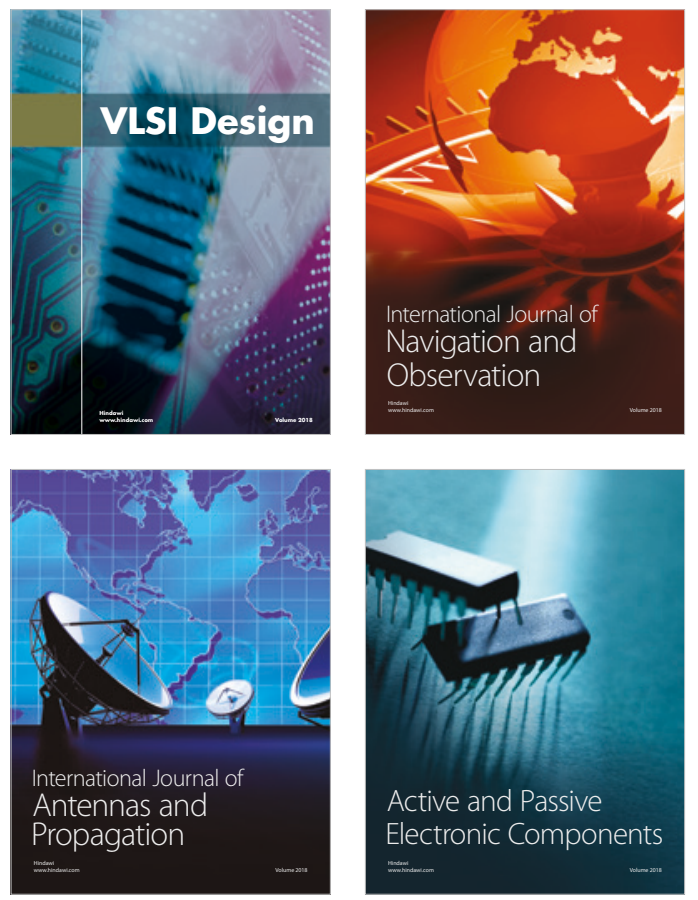
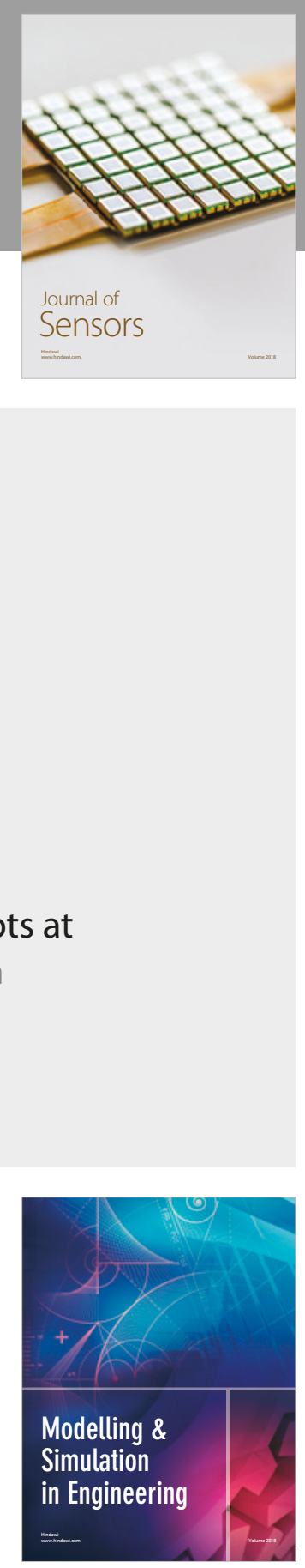

\section{Advances \\ Multimedia}
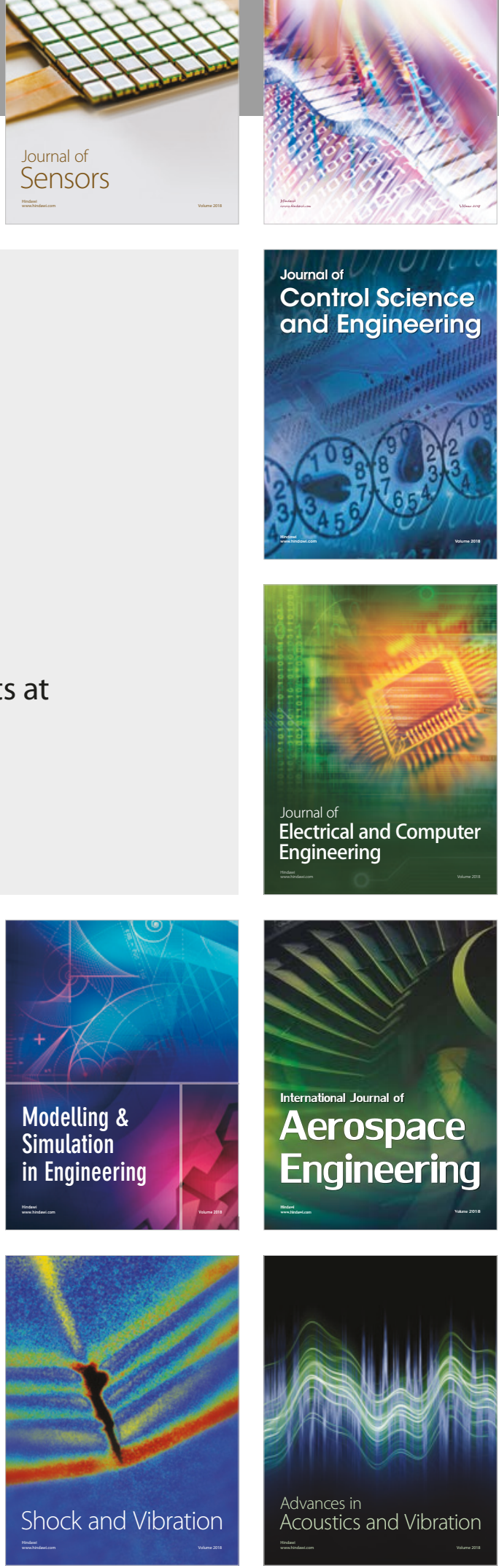